\title{
Downscaling Dinâmico através do Modelo RegCM para Diferentes Inicializações Utilizando Dados do CFSv2
}

\author{
Ismael Guidson Farias de Freitas ${ }^{1}$ (D), Helber Barros Gomes ${ }^{1,2}$, Glauber Lopes Mariano ${ }^{2}$, \\ Maria Cristina Lemos da Silva ${ }^{2}$, Matheus José Arruda Lyra ${ }^{1}$, Thayna Silva Vila Nova ${ }^{2}$, \\ Fabrício Daniel dos Santos Silva ${ }^{2}$, Laurizio Emanuel Ribeiro ${ }^{3}$, Cristiano Prestrelo de Oliveira ${ }^{4}$ \\ ${ }^{1}$ Unidade Acadêmica de Ciências Atmosféricas, Universidade Federal de Campina Grande, \\ Campina Grande, PB, Brasil. \\ ${ }^{2}$ Instituto de Ciências Atmosféricas, Universidade Federal de Alagoas, Maceió, AL, Brasil. \\ ${ }^{3}$ Centro de Previsão de Tempo e Estudos Climáticos, Instituto Nacional de Pesquisas Espaciais, \\ Cachoeira Paulista, SP, Brasil. \\ ${ }^{4}$ Departamento de Ciências Atmosféricas e Climáticas, Universidade Federal do Rio Grande do \\ Norte, Natal, RN, Brasil.
}

Recebido em: 27 de Junho de 2020 - Aceito em: 12 de Agosto de 2020

\begin{abstract}
Resumo
O objetivo deste trabalho é avaliar as previsões climáticas regionais de precipitação sobre o Brasil durante a estação do inverno de 2018 através do modelo RegCM4.7 com diferentes inicializações, tanto espacial como em 5 áreas específicas. Para realizar alertas de possíveis anomalias abaixo/acima da normal climatológica, é necessário verificar a habilidade destes modelos em prever de forma antecipada a precipitação. O modelo RegCM4.7 foi conduzido com dados do modelo Global Climate Forecast System Version 2. As destrezas das previsões foram avaliadas de forma qualitativa e quantitativa, comparando resultados com os dados do Climate Prediction Center (CPC). Os resultados mostraram que o RegCM4.7 conseguiu prever de forma coerente a precipitação com alguns meses de antecedência para o trimestre junho, julho e agosto (JJA), com menores erros sobre as regiões Nordeste e Sudeste do Brasil, onde maiores erros foram identificados sobre os subdomínios AMZ e SUL. Observou-se que as correlações das previsões foram inferiores a 0,8 durante todos os experimentos e subdomínios, exceto para região do Nordeste que apresentou os maiores valores de correlação. De maneira geral, destaca-se que o modelo foi hábil em prever a distribuição espacial de precipitação com antecedência sobre todo domínio, porém com tendência de subestimar o observado.
\end{abstract}

Palavras-chave: RegCM, CFSv2, previsão climática, downscaling dinâmico.

\section{Dynamic Downscaling Using the RegCM Model for Different Initializations Using CFSv2 Data}

\begin{abstract}
The main objective of this study was to evaluate the regional climate forecasts of precipitation over Brazil during the winter season of 2018 through the RegCM4.7 model. It was used with different initializations, both spatial and in five specific areas. To emit alerts of possible below/above climate normal anomalies is necessary the verification these model abilities in predicting precipitation. The RegCM4.7 model was used with Global Climate Forecast System Version 2 global model data. The forecast quality was evaluated qualitatively and quantitatively, comparing its results with Climate Prediction Center (CPC) analysis data. The RegCM4.7 model was able to predict precipitation consistently a few months in advance for the june, july and august quarter (JJA), with minor mistakes over the northeastern and southeastern Brazil. However, the biggest errors were identified over the northern and southern regions. Prediction correlations were less than 0.8 during every experiments and subdomains, except for the Northeast region that presented the highest correlation values. In general, it stands out that RegCM4.7 was able to predict the spatial distribution of precipitation in advance over every domain, but with a tendency to underestimate what was observed.
\end{abstract}

Keywords: RegCM, CFSv2, weather forecast, dynamic downscaling. 


\section{Introdução}

O Brasil localiza-se entre as latitudes de $5^{\circ} \mathrm{N}$ e $33^{\circ}$ $\mathrm{S}$, abrangendo baixas e altas latitudes, e possuindo grande extensão territorial. Assim, diversos fenômenos atmosféricos atuam sob esta região, influenciando diretamente a variabilidade da precipitação que resulta numa heterogeneidade climática (Reboita et al., 2010). Desta forma, é importante conhecer e prever os padrões pluviométricos climáticos em todo país. Essas previsões são de suma importância para o planejamento e tomadas de decisões futuras para as atividades humanas, particularmente aquelas ligadas à agricultura, geração de energia hidrelétrica, transportes e etc (Giorgi e Mearns, 1999; Alves et al., 2009).

Vários esforços estão sendo realizados para melhorar as previsões climáticas regionais ao redor do mundo através do COordinated Regional climate Downscaling EXperiment (CORDEX), que foi iniciado em 2008 como resultado da força tarefa no downscaling climático regional, formado pelo World Climate Research Program (WCRP) (Jesus, 2014; Ozturk et al., 2018; Fantini et al., 2018). No Brasil, diversos estudos analisaram o desempenho das previsões climáticas utilizando diferentes modelos climáticos regionais (Ávila et al., 2013; Souza, 2016 e Llopart et al. 2017; Reboita et al., 2018; Araújo et al., 2019). Os Modelos Climáticos Regionais (MCRs) são ferramentas fundamentais para estudar questões científicas relacionadas à variabilidade, mudanças e impactos do clima em escalas regionais (Ozturk et al., 2018; Oh e Suh, 2018). Neste sentido, existem algumas vantagens na utilização dos MCRs, como por exemplo sua relativa economia computacional em relação aos modelos globais, pois seu uso pode ser realizado a partir de computadores com configurações simples, além da representação de forma mais detalhada das características regionais de topografia e superfície (Alves et al., 2007).

Apesar das vantagens nas previsões climáticas mensais, sub-sazonais e sazonais, os MCRs possuem algumas limitações em reproduzir alguns processos físicos na atmosfera, como a convecção profunda. Assim, como o Brasil apresenta uma grande dimensão continental e diferentes características biogeofísicas (Vanzolini, 1992; Prado, 2003; Mendonça, et al., 2014), é importante avaliar a destreza de suas previsões em regiões distintas, para que possa servir de auxílio para a tomada de decisões, desenvolvimento de políticas públicas e etc.

O Regional Climate Model (RegCM) além de ser um dos MCRs que compõem o experimento CORDEX, é muito utilizado em estudos de previsões mensais, subsazonais e sazonais, sob diferentes regiões do globo (Ozturk et al., 2018; Oh e Suh, 2018; Amador et al., 2018; Chung et al., 2018), com resolução horizontal de dezenas de quilômetros e capacidade de reproduzir processos com diferentes tipos de cobertura da superfície e delimitações entre oceano e continente. Avaliar as previsões climáticas realizadas pelo modelo RegCM, torna-se importante, uma vez que podem auxiliar como ferramenta para prever anomalias extremas de precipitação, sejam elas abaixo ou acima da normal climatológica (Gonçalves, 2015).

Diante do exposto, este trabalho tem como objetivo avaliar as previsões climáticas regionais de precipitação sobre o Brasil para o trimestre junho, julho e agosto (estação de inverno) de 2018, utilizando a versão 4.7 do modelo RegCM com diferentes inicializações, assim como investigar a sua habilidade em prever com alguns meses de antecedência a distribuição de precipitação em quatro subdomínios específicos, utilizando técnicas estatísticas.

\section{Materiais e Métodos}

\subsection{Dados}

As previsões climáticas do modelo climático regional (RegCM) foram iniciadas e os contornos atualizados com dados do modelo Global Climate Forecast System Version 2 (CFSv2; Saha et al., 2014) do National Centers for Environmental Prediction (NCEP). O CFSv2 é um modelo integrado diariamente em uma grade de $0,9^{\circ} \mathrm{x}$ $0,9^{\circ}$ de latitude e longitude com 64 níveis na vertical, disponíveis a cada 5 dias, e com resolução temporal de 6 horas $(0000,0600,1200$ e 1800 UTC), uma descrição mais detalhada é apresentada em Saha et al. (2014).

Para avaliar a destreza das previsões, de forma qualitativa e quantitativa, foram utilizados dados de análises do Climate Prediction Center (CPC) (NCAR, 2018). As análises do CPC consistem em dados diários e disponibilizados com resolução de $0,5^{\circ} \times 0,5^{\circ}$ de latitude/longitude. Estes dados estão disponíveis de 1979 até o presente e descrevem as características espaciais da precipitação apenas sobre áreas continentais.

\subsection{Modelo RegCM}

Este estudo utilizou a versão 4.7 do Modelo Climático Regional (a partir daqui RegCM4.7), que foi originalmente desenvolvido na década de 1980 no National Center for Atmospheric Research (NCAR) por Dickinson et al. (1989) e atualmente encontra-se na versão 4.7 (Giorgi e Elguindi, 2014). O RegCM teve como base o modelo atmosférico Mesoscale Model version 4 (MM4; Anthes et al., 1987), que trata de um modelo comunitário de área limitada em coordenada vertical sigma-p e grade horizontal B de Arakawa, com as equações primitivas para fluido compressível e hidrostático (Souza et al., 2016). Foi utilizado o esquema de superfície Biosphere-Atmosphere Transfer Scheme (BATS; Dickinson et al., 1993), o esquema de transferência radiativa do Community Climate Model (CCM3; Kiehl et al., 1996) e esquema de convecção Emanuel (Emanuel, 1991) para resolver a parametrização cumulus. Todos os esquemas foram utilizados 
com base em outros estudos, que identificaram o bom desempenho das parametrizações na região de estudo (Silva, 2006; Gonçalves, 2015; Llopart et al., 2017).

\subsection{Design dos experimentos}

As previsões climáticas foram realizadas a partir dos meses de fevereiro a junho de 2018 para prever a estação de inverno austral subsequente (Junho-Julho-Agosto, JJA). A escolha desse período (trimestre JJA de 2018) se deve a um padrão anômalo de precipitação observado (descrito na secção 2.5) sobre todas as regiões do Brasil. O período de integração foi de 7 meses, no entanto, as análises foram realizadas para o trimestre junho, julho e agosto (JJA) de 2018, e também os meses que antecederam as previsões para o cálculo do RMSE diário (raiz do erro quadrático médio), o que possibilita verificar crescimentos de erros nas previsões. Todas as previsões foram iniciadas às $0000 \mathrm{UTC}$ do dia $1^{\circ}$ de cada mês, conforme descrito na Tabela 1, que também mostra o layout dos experimentos numéricos realizados para prever o inverno de 2018 (em destaque em cor na tabela).

A área de integração do modelo está compreendida entre as latitudes de $10^{\circ} \mathrm{N}-40^{\circ} \mathrm{S}$ e longitude de $35^{\circ} \mathrm{W}$ $75^{\circ} \mathrm{W}$, cobrindo praticamente toda América do Sul e parte do oceano Atlântico (Fig. 1). As resoluções horizontais e verticais foram de $50 \mathrm{~km}$ e 23 níveis sigma-pressão, respectivamente, com topo em $50 \mathrm{hPa}$.

Na Fig. 1 observa-se também todos os subdomínios selecionados para avaliar a destreza do modelo espacialmente através de médias mensais e cálculos do erro médio (BIAS), e regionalmente a amplitude da raiz do erro quadrático médio (RMSE). A Tabela 2 mostra as dimensões (latitudes e longitudes) de todos os subdomínios avaliados. Alguns destes subdomínios foram avaliados em estudos prévios por outros autores, porém com dimensões diferentes (Silva, 2006, Gomes, 2012, Dantas et al., 2013; Gonçalves, 2015; Dias, 2017; Reboita et al., 2018).

\subsection{Validação das previsões}

Conforme já mencionado acima, para avaliar a destreza da precipitação prevista pelo modelo RegCM4.7 de

Tabela 1 - Design dos 5 experimentos numéricos realizados com o modelo RegCM4.7, onde alterou-se as datas de inicialização de acordo com a disponibilidade dos dados do CFSv2.

\begin{tabular}{ccccccc}
\hline \multicolumn{7}{c}{2018} \\
\hline Fev & Mar & Abr & Mai & Jun & Jul & Ago \\
\hline $\mathrm{X}$ & $\mathrm{X}$ & $\mathrm{X}$ & $\mathrm{X}$ & $\mathrm{X}$ & $\mathrm{X}$ & $\mathrm{X}$ \\
& $\mathrm{X}$ & $\mathrm{X}$ & $\mathrm{X}$ & $\mathrm{X}$ & $\mathrm{X}$ & $\mathrm{X}$ \\
& & $\mathrm{X}$ & $\mathrm{X}$ & $\mathrm{X}$ & $\mathrm{X}$ & $\mathrm{X}$ \\
& & $\mathrm{X}$ & $\mathrm{X}$ & $\mathrm{X}$ & $\mathrm{X}$ \\
& & & $\mathrm{X}$ & $\mathrm{X}$ & $\mathrm{X}$
\end{tabular}

$\mathrm{X}$ : meses de previsão e inicialização do modelo; em destaque o período de interesse.

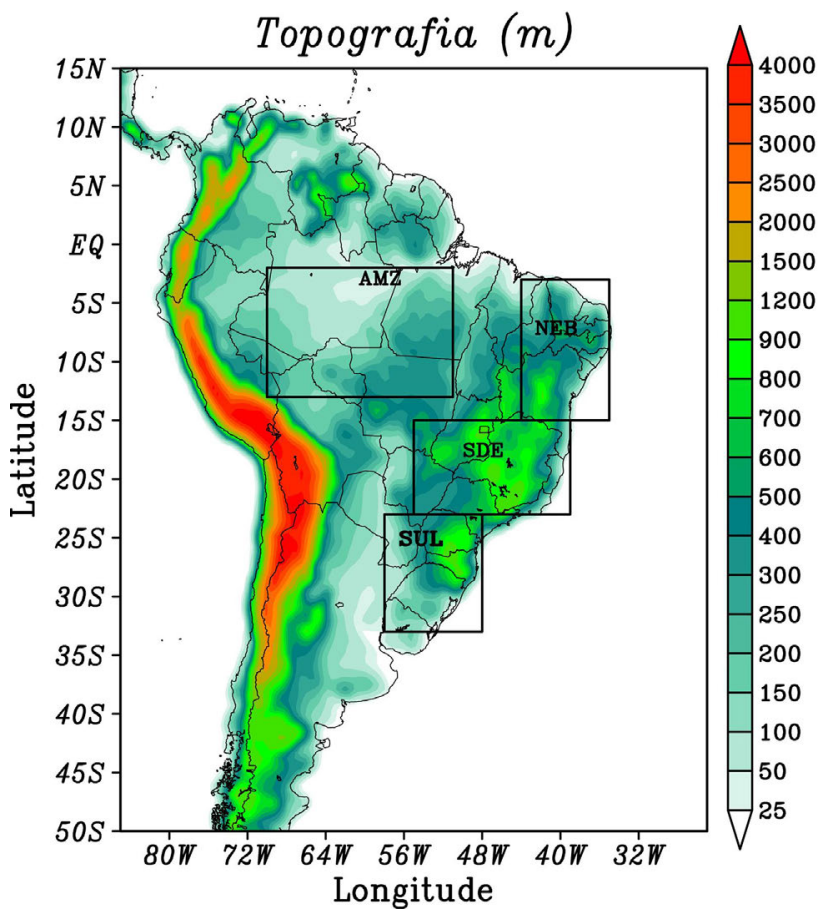

Figura 1 - Domínio e subdomínios da área de estudo utilizado pelo RegCM4.7. As cores representam a topografia (m) da região.

forma quantitativa, foram calculados espacialmente as médias do trimestre e o Erro Médio (BIAS), e regionalmente a amplitude média da raiz do erro quadrático médio (RMSE) em cada subdomínio (descrito na Tabela 2). A descrição dos índices estatísticos supracitados, é apresentado abaixo (Wilks, 2011).

O BIAS, considera uma determinada variável $(X)$ com um sub-índice $(M)$, indicando o valor simulado $\left(X_{M}\right)$ e o valor observado $\mathrm{O}\left(X_{O}\right)$. Assim, o BIAS para a variável de interesse $(X)$, foi calculado para determinada área da seguinte forma (Eq. (1)):

$$
b=\frac{1}{N} \sum_{i=1}^{N}\left(X_{M i}-X_{O i}\right)
$$

no qual $i$ representa o índice do ponto de grade e $N$ o número total de pontos avaliados.

O RMSE mede a dimensão dos erros, calculado de acordo com a Eq. (2):

Tabela 2 - Latitudes e Longitudes dos subdomínios avaliados.

\begin{tabular}{lcc}
\hline Subdomínios & Latitudes & Longitudes \\
\hline Amazônia e Centro-Oeste - AMZ & $2^{\circ} \mathrm{S}-13^{\circ} \mathrm{S}$ & $51^{\circ} \mathrm{W}-70^{\circ} \mathrm{W}$ \\
Nordeste-NEB & $3^{\circ} \mathrm{S}-15^{\circ} \mathrm{S}$ & $35^{\circ} \mathrm{W}-44^{\circ} \mathrm{W}$ \\
Sudeste-SDE & $15^{\circ} \mathrm{S}-23^{\circ} \mathrm{S}$ & $39^{\circ} \mathrm{W}-59^{\circ} \mathrm{W}$ \\
Sul-SUL & $23^{\circ} \mathrm{S}-33^{\circ} \mathrm{S}$ & $48^{\circ} \mathrm{W}-58^{\circ} \mathrm{W}$ \\
\hline
\end{tabular}




$$
R M S E=\sqrt{\frac{1}{N} \sum_{i=1}^{N}\left(X_{M i}-X_{0 i}\right)}
$$

Foram calculados ainda, o coeficiente de correlação de Pearson e Desvio padrão para serem dispostos no diagrama de Taylor. Este diagrama foi elaborado com a finalidade de fornecer um resumo estatístico conciso de quão bem ou mau os padrões se combinam em termos de correlação e desvio padrão. Esta análise compara valores previstos e observados, com o dado observado utilizado como referência. Uma descrição mais detalhada de como calcular tais diagramas podem ser obtida em Taylor (2001).

\subsection{Inverno de 2018}

Através de uma análise de variabilidade nos dados das estações meteorológicas do Instituto Nacional de Meteorologia (http://inmet.gov.br/portal/index.php? $\mathrm{r}=$ clima/desvioChuvaTrimestral), observou-se que durante o trimestre JJA de 2018 a precipitação apresentou padrões anômalos sobre os quatro subdomínios avaliados (INMET, 2020). Foram observados déficits entre $20-500 \mathrm{~mm}$ no Nordeste e em parte da região Norte e Centro-oeste, superávit entre 20-200 $\mathrm{mm}$ no Sudeste, enquanto na região Sul em partes déficits $(50-400 \mathrm{~mm})$ e outras superávits (20-100 mm). Desta forma, foi avaliado a habilidade do modelo RegCM4.7 em prever essa precipitação anômala com até quatro meses de antecedência.

\section{Resultados e Discussão}

A Fig 2 mostra as previsões climáticas de precipitação para o trimestre de JJA de 2018 (Fig. 2. a1-5), análises do CPC (Fig. 2. b1-5) e bias (Fig. 2. c1-5). De maneira geral, observa-se que o modelo RegCM4.7 foi hábil em prever a intensidade da precipitação nas previsões iniciadas nos meses de abril a junho sobre praticamente todo domínio, bem como os padrões espaciais associados, principalmente sobre as regiões com baixa quantidade de precipitação. Ressalta-se ainda que não houve melhorias nas previsões à medida que as suas inicializações se aproximaram do trimestre de estudo, como por exemplo, inicialização da previsão com um mês de antecedência.

Os maiores desvios foram observados sobre os subdomínios AMZ e SUL, onde os totais de precipitação foram mais intensos (Fig. 2, coluna direita). Por outro lado, nas demais regiões o RegCM4.7 apresentou menores amplitudes no bias. De acordo com Reboita et al. (2010), durante o trimestre JJA os modelos conseguem prever com alguns meses de antecedência a precipitação de forma coerente sobre a região Sudeste do Brasil (estação seca). Destaca-se também que a distribuição espacial de precipitação observada (Fig. 2, coluna central) sobre a região central da América do Sul, sentido noroeste-sudeste, foi bem representada pelo modelo (Fig. 2, coluna esquerda). De maneira geral, o modelo RegCM4.7 subestimou (superestimou) a precipitação observada sobre os extremos norte e sul (costa leste, exceto a região Sul) do Brasil em todos os meses que antecedem a estação de interesse (JJA). Gonçalves (2015) através de previsões climáticas sazonais com o modelo RegCM durante o período de agosto/2013 a agosto/2014 para região Sul do Brasil, documentou que o modelo subestimou a precipitação prevista sobre esta região, sendo confirmado com estes resultados.

A Fig. 3 apresenta a variação temporal diária do RMSE para precipitação nos quatro subdomínios para os 5 experimentos com diferentes inicializações do modelo RegCM4.7. Nota-se na Fig. 3 que o RMSE apresentou valores mais elevados nos primeiros dias de previsão em todos os experimentos, principalmente nos subdomínios AMZ e NEB, isso pode ser explicado devido a mudança repentina nas condições dinâmica da atmosfera (Zhong et al., 2009), uma vez que não se utilizou período de spinup para calcular o RMSE diário. O subdomínio SUL mostra valores mais expressivos de RMSE em todo período de previsão devido a subestimativa da precipitação nesta região, independente do mês de inicialização do modelo.

Verifica-se na inicialização de fevereiro em todos os subdomínios (Fig. 3) que o modelo apresenta picos significativos de até $23 \mathrm{~mm}$ no RMSE durante os meses de previsão. De acordo com dados do GGWEATHER (2020) durante este período de inicialização, anomalias de temperatura da superfície do pacífico indicavam a existência de La Niña (Oceanic Niño Index: -0,9) o que mostra a capacidade do modelo em reproduzir as precipitações associadas a este evento meteorológico. Desta forma, uma provável hipótese a respeito da boa sensibilidade do modelo é que durante este evento de La Niña a previsão reproduziu bem as influências da temperatura da superfície do mar no pacífico, uma vez que foram observadas superestimativas de precipitação no subdomínio NEB e boa previsibilidade no SDE e SUL (Fig. 3).

Na Fig. 3-a e b, nota-se que nas inicializações a partir de março, o modelo mostra-se eficiente entre os dias 01 de junho e 31 de agosto de 2018, onde apresenta menor amplitude nos valores de RMSE. No subdomínio SDE (Figura 3-b) observa-se que os maiores picos acontecem dentro do trimestre JJA, o que resultou em fortes superestimativas de precipitação. Na região SUL (Fig. 3-c), observa-se que os picos de RMSE foram intensos em todas as inicializações do modelo, principalmente nas previsões iniciadas entre março e junho de 2018, onde o modelo subestima a precipitação com maior intensidade.

A Fig. 4 apresenta o diagrama de Taylor que dispõe um resumo de ferramentas estatísticas como desvio-padrão e correlação de forma visual. Este diagrama é apresentado resultados para os quatro subdomínios avaliados e as cinco inicializações. Pode-se observar que os subdomínios SDE e NDE apresentaram maior concordância com os observados, com maiores valores de correlação e desvio padrão mais próximos ao observado, sendo a previsão ini- 

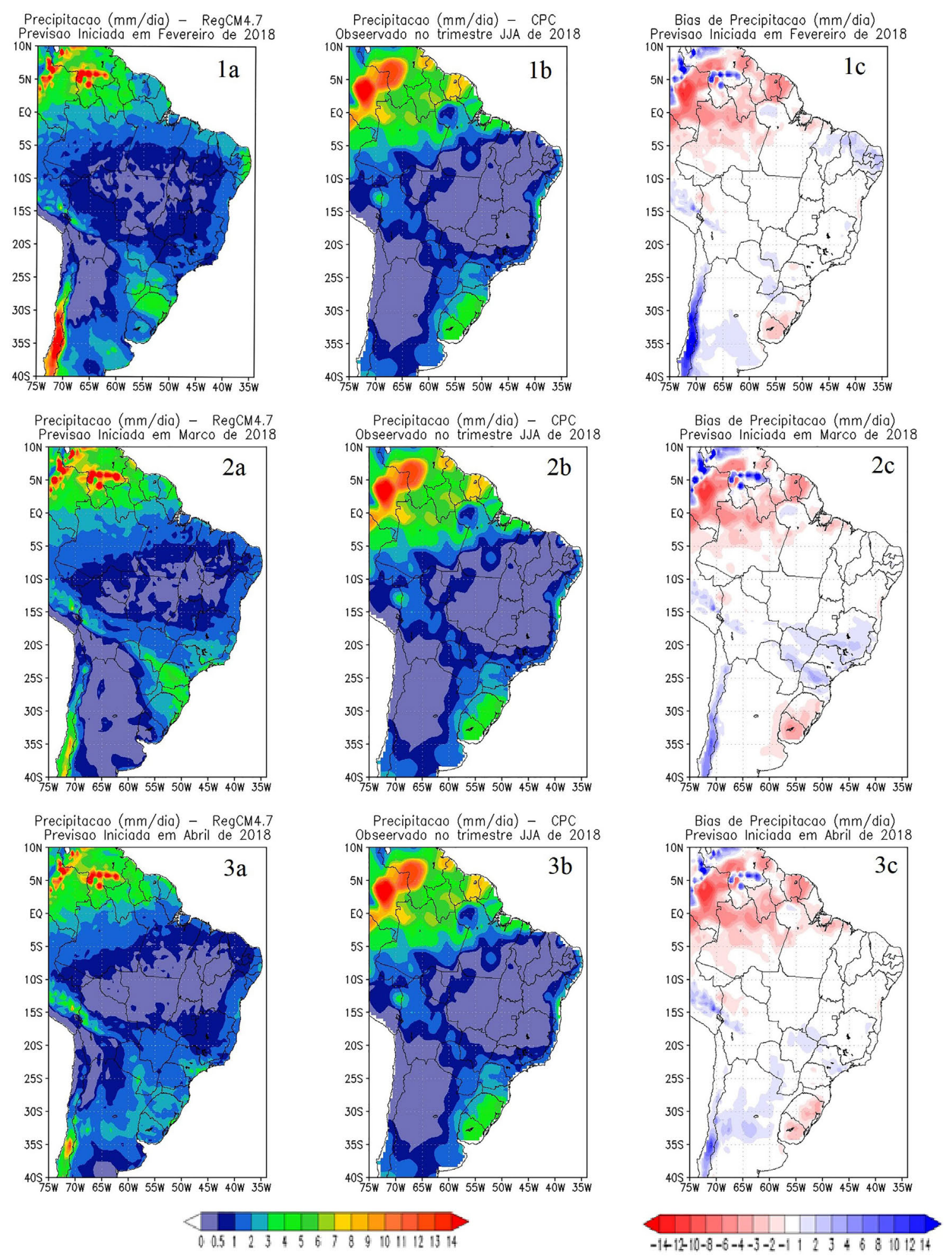

Figura 2 - Precipitação média (mm/dia) para o trimestre JJA de 2018: (a, coluna esquerda) previsões utilizando diferentes inicializações do modelo RegCM4.7, (b, coluna central) observado pelo CPC e (c, coluna direita) bias de precipitação. Por questões de visualização a coluna central foi disposta sempre com a mesma figura.

cializada em fevereiro a que obteve melhor resultado de correlação na região SDE (aproximadamente 0,6 ) e as previsões inicializadas em março, abril e junho na região
NDE (entre 0,6-0,8). No NDE da previsão iniciada em julho apresentar maior correlação $(0,8)$, o desvio padrão das previsões inicializadas em março e abril foram mais 

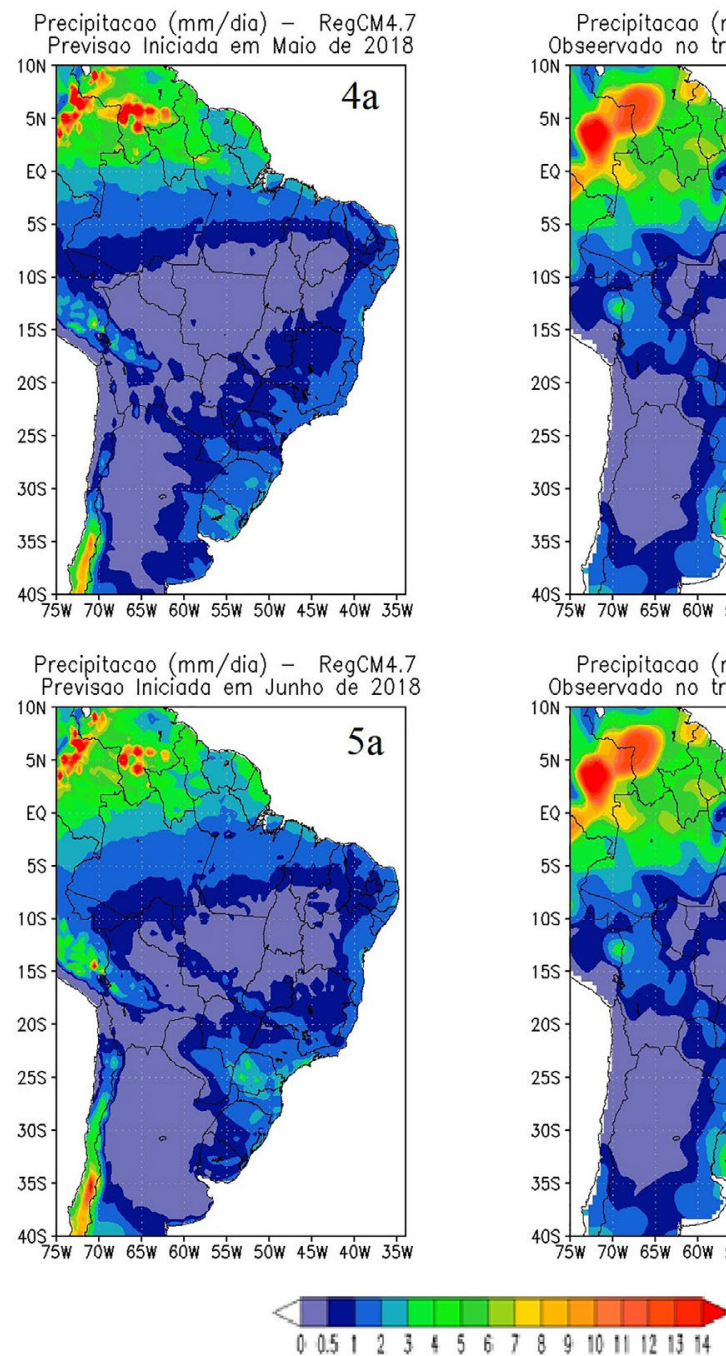

Precipitacao $(\mathrm{mm} / \mathrm{dia})-\mathrm{CPC}$ Obseervado no trimestre JJA de 2018

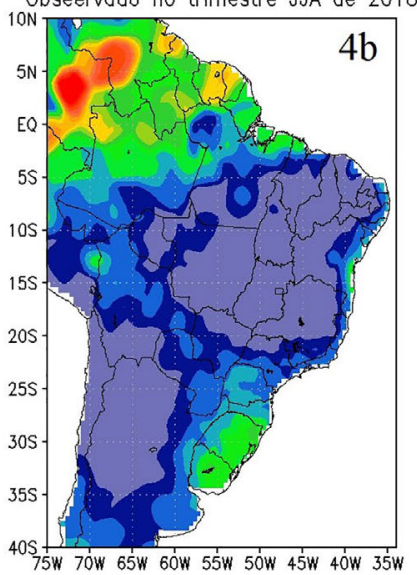

Precipitacao ( $\mathrm{mm} / \mathrm{dia})-\mathrm{CPC}$ Obseervado no trimestre JJA de 2018

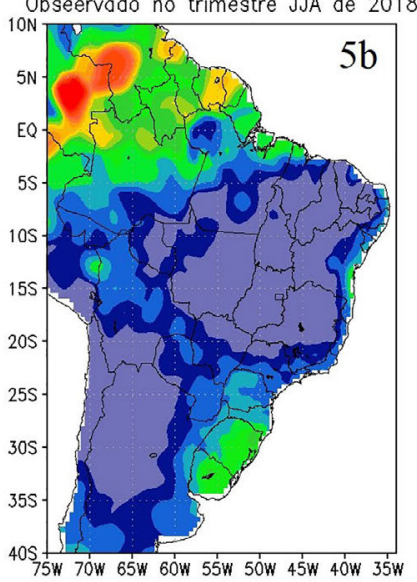

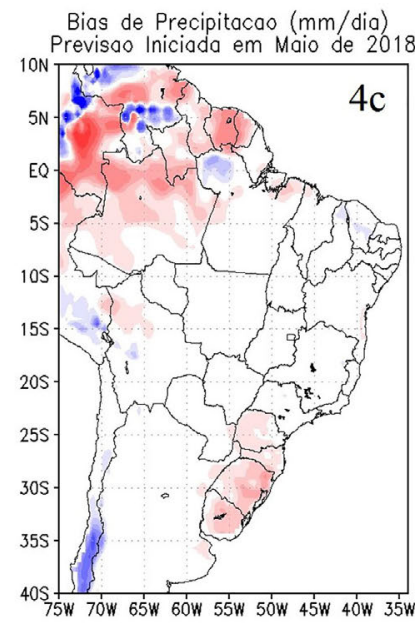
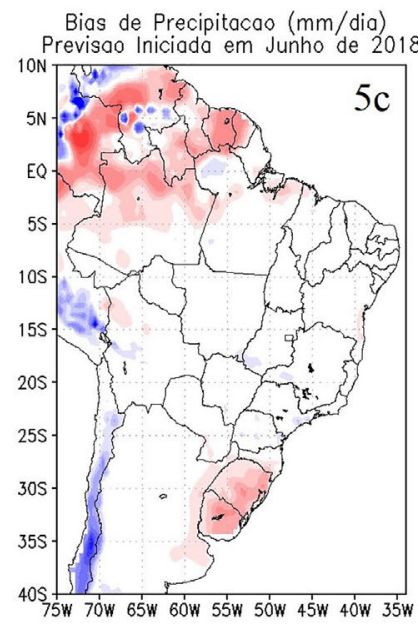

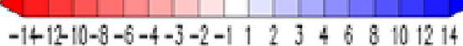

Figura 2 (cont.) - Precipitação média (mm/dia) para o trimestre JJA de 2018: (a, coluna esquerda) previsões utilizando diferentes inicializações do modelo RegCM4.7, (b, coluna central) observado pelo CPC e (c, coluna direita) bias de precipitação. Por questões de visualização a coluna central foi disposta sempre com a mesma figura.

próximo ao observado. Nota-se ainda que nos subdomínios AMZ e SUL todos os experimentos apresentaram correlações abaixo de 0,5 e desvios bastante divergentes dos valores de referência, onde variou entre 2,25-4,25 no SUL (referência 3,50), enquanto no AMZ foram entre 0,30-0,54 (referência 1,75), em ambos os subdomínios a previsão inicializada em fevereiro apresentou melhores resultados. De acordo com estes resultados de correlação e desvio padrão, observa-se uma maior habilidade do modelo em prever a precipitação do trimestre JJA para os subdomínios SDE e NDE.

\section{Considerações Finais}

Neste estudo, foram realizadas 5 inicializações numéricas, onde foi alterado o mês de inicialização do modelo para verificar a habilidade do modelo RegCM4.7 em prever com antecedência a precipitação do inverno austral de 2018, comparando as previsões com dados do CPC. Além disso, verificar através do RMSE diário, possíveis associações de erros, antes do período a ser previsto.

De acordo com os resultados, durante o trimestre JJA de 2018, verificou-se que os padrões de precipitação menos intensos foram previstos de forma coerente, quando comparados com o CPC, como por exemplo no Nordeste e Sudeste, enquanto as precipitações mais intensas como a região Sul e parte do Norte do Brasil o modelo apresenta pouca habilidade. Desta forma através do RMSE diário, constatou-se que erros das previsões utilizando diferentes inicializações variaram em função do tempo e região, onde em muitos casos apresentaram pouca/muita habilidade em realizar as previsões, revelando erros antes do período de interesse.

Embora as análises apresentem limitação por ter apenas um trimestre, devido a deficiência computacional 

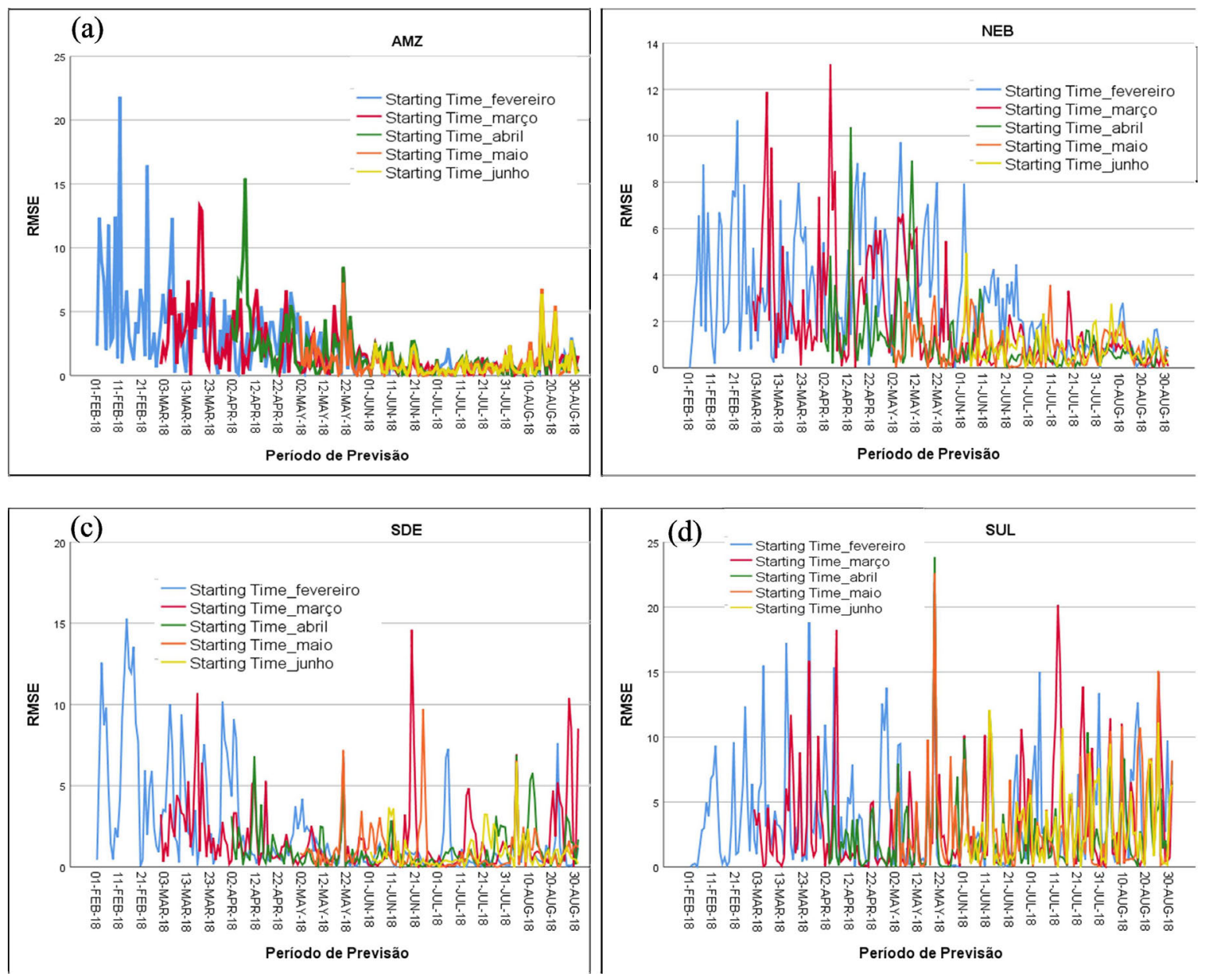

Figura 3 - Variação temporal diária da raiz do erro quadrático médio (RMSE) nos subdomínios AMZ (a), NEB (b) SDE (c) e SUL (d) a partir do início de todas as inicializações.

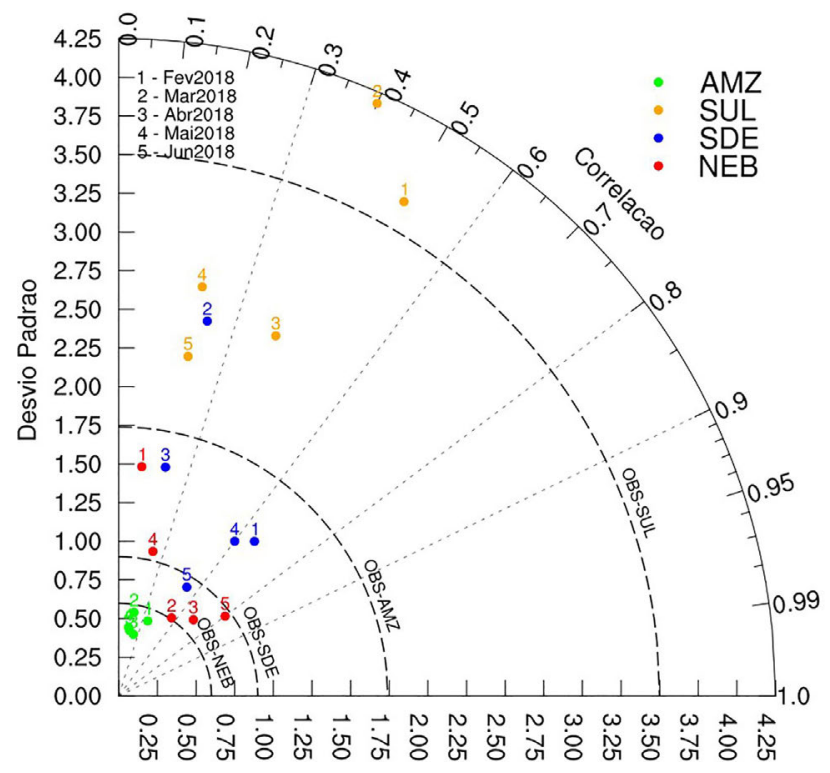

Figura 4 - Diagrama de Taylor com valores de correlação e desvio padrão para os subdomínios AMZ, NEB, SDE e SUL para todas as inicializações. Onde OBS é referência calculada com os dados observados para todos os subdomínios. 
para realizar e salvar as previsões, nota-se que o modelo consegue prever de forma antecipada alguns eventos de precipitação, principalmente nas regiões Nordeste, Sudeste e Sul. Posteriormente pretende-se realizar as configurações computacionais necessárias para fazer previsões para outros períodos e poder gerar análises através da climatologia do modelo RegCM4.7.

\section{Referências}

ALVES, L.M. Simulações da Variabilidade do Clima Presente sobre a América do Sul utilizando um Modelo climático Regional. Tese de Doutorado, Programa de Pós-Graduação em Meteorologia, Instituto Nacional de Pesquisas Espaciais, Cachoeira Paulista, 2007.

ALVES, L.M.; CHOU, S-C.; MARENGO, J.A. Avaliação das previsões de chuvas sazonais do modelo ETA climático sobre o Brasil. In: XIII Congresso Brasileiro de Meteorologia, Fortaleza: SBMET, 2009.

AMADOR, J.A.; AMBRIZZI, T.; ARRITT, R.W.; CASTRO, C.L.; CAVAZOS, T.; MOTA, R.C.; FRANCO, R.F.; GIORGI, F.; GUILANI, G.; LEE, H.; PEREZ, M.M.; RIVERA, E.R. Putting into action the REGCM4. 6 regional climate model for the study of climate change, variability and modeling over Central America and Mexico. Atmósfera, v. 31, n. 2, p. 185-188, 2018.

ANTHES, R.A.; HSIE, E.-Y.; KUO, Y.H. Description of the Penn State/NCAR Mesoscale Model Version 4 (MM4). Boulder: NCAR, 1987.

ARAÚJO, G.; SILVA, C.; SILVA, A.G. Avaliação das parametrizações cumulos Emanuel e Grell do modelo climático regional RegCM4: Simulando a precipitação e temperatura a superfície sobre o Nordeste brasileiro durante o outono austral. Anuário do Instituto de Geociências, v. 42, n. 1, p. 231-240, 2019.

ÁVILA, P.L.R.; SOUZA, E.B.; PINHEIRO, F. Simulação da precipitação sazonal com o Regcm4 sobre o estado do Pará para anos de El Niño e La Niña. Revista Brasileira de Geografia Física, v. 6, n. 5, p. 1316-1335, 2013.

CHUNG, J.X.; JUNENG, L.; TANGANG, F.; JAMALUDDIN, A.F. Performances of BATS and CLM land-surface schemes in RegCM4 in simulating precipitation over CORDEX Southeast Asia domain. International Journal of Climatology, v. 38, n. 2, p. 794-810, 2018.

DANTAS, V.A.; AMORIM, A.C.B.; COSTA, M.S.; SILVA, C.M.S. Downscaling dinâmico sobre o Nordeste do Brasil utilizando um modelo climático regional: Impacto de diferentes parametrizações na precipitação simulada. Revista Brasileira de Geografia Física, v. 6, n. 4, p. 995-1008, 2013.

DIAS, C.G. Validação de Previsões Climáticas Sazonais Sobre o Brasil: Modelos Globais e Regional. Dissertação de Mestrado, Programa de Pós Graduação em Meteorologia, Universidade Federal de Itajubá, Itajubá, 2017.

DICKINSON, R.E.; ERRICO, R.M.; GIORGI, F.; BATES, G.T. A regional climate model for the western United States. Climatic change, v. 15, n. 3, p. 383-422, 1989.

DICKINSON, R.E.; HENDERSON-SELLERS, A.; KENNEDY, P.J. Biosphere-Atmosphere Transfer Scheme (BATS) ver- sion $1 \mathrm{E}$ as coupled to the NCAR Community Climate Model. NCAR Technical Report. TN-387+STR, NCAR, Boulder: NCAR, 72 p., 1993.

EMANUEL, K.A. A scheme for representing cumulus convection in large-scale models. Journal of the Atmospheric Sciences, v. 48, n. 21, p. 2313-2329, 1991.

FANTINI, A.; COPPOLA, E.; VERDECCHIA, M. Evaluation of two new high-resolution EURO-CORDEX RegCM simulations with a new Italian precipitation dataset. In: EGU General Assembly Conference Abstracts. Vienna: EGU, 2018.

GGWEATHER. El Niño and La Niña Years and Intensities, disponível em http:/ggweather.com/enso/oni.htm, acesso em 3 de fevereiro de 2020, 2020 .

GIORGI, F.; ELGUINDI, N. Regional Climatic Model RegCM User's Guide Version 4.4. Trieste, Italy, 2014.

GIORGI, F.; MEARNS, L.O. Introduction to special section: Regional climate modeling revisited. Journal of Geophysical Research: Atmospheres, v. 104, n. D6, p. 63356352, 1999.

GOMES, H.B. Distúrbios Ondulatórios de Leste no Nordeste Brasileiro: Climatologia e Modelagem Numérica. Tese de Doutorado, Programa de Pós Graduação em Meteorologia, Universidade de São Paulo, São Paulo, 2012.

GONÇALVES, J.S. Avaliação da Previsão Numérica Sazonal de Precipitação para o Rio Grande do Sul. Dissertação de Mestrado, Programa de Pós-Graduação em Meteorologia, Universidade Federal de Santa Maria, Santa Maria, 2015.

Instituto Nacional de Meteorologia do Brasil. Desvio de Precipitação Trimestral no Brasil, disponível em http://www. inmet.gov.br/portal/index.php? $\mathrm{r}=$ clima/desvioChuvaTrimestral, acesso em 3 de fevereiro de 2018, 2020.

JESUS, E.M. Simulações Climáticas Regionais Para o CORDEX Sobre a América do Sul e Impactos das Frentes Frias na Climatologia Simulada. Tese de Doutorado, Programa de Pós-Graduação em Meteorologia, Universidade de São Paulo, São Paulo, 2016

KIEHL, J.T.; HACK, J.J.; BONAN, G.B.; BOVILLE, B.A. Description of the NCAR Community Climate Model (CCM3). Boulder, Colorado: NCAR Technical Note, NCAR/TN-420+STR, 1996.

LLOPART, M;; ROCHA, R.P.; REBOITA, M.; CUADRA, S. Sensitivity of simulated South America climate to the land surface schemes in RegCM4. Climate Dynamics, v. 49, n. 11-12, p. 3975-3987, 2017.

MENDONÇA, M.; ROMERO, H.; OPAZO, D. Análise multiescalar para a comprenssao de causas e consequencias da variabilidade climática na América do Sul. In: SILVA, C.A.; FIALHO, E.S.; STEINKE, E.T. Experimentos em Climatología Geográfica. São Paulo: Asociación Brasileña de Climatología Geográfica, p. 271-290, 2014.

NATIONAL CENTER FOR ATMOSPHERIC RESEARCH STAFF. The Climate Data Guide: CPC Unified GaugeBased Analysis of Global Daily Precipitation. Retrieved from https://climatedataguide.ucar.edu/climate-data/cpcunified-gauge-based-analysis-global-daily-precipitation, acesso entre 1 de março de 2018, 2018.

$\mathrm{OH}, \mathrm{S}-\mathrm{G}$.; SUH, M-S. Changes in seasonal and diurnal precipitation types during summer over South Korea in the late 
twenty-first century (2081-2100) projected by the RegCM4. 0 based on four RCP scenarios. Climate Dynamics, v. 51, n. 7, p. 3041-3060, 2018.

OZTURK, T.; TURP, M.T.; TURKES, M.; KURNAZ, M. Future projections of temperature and precipitation climatology for CORDEX-MENA domain using RegCM4. 4. Atmospheric Research, v. 206, n. 1, p. 87-107, 2018.

PRADO, D.E. As caatingas da América do Sul. Ecologia e Conservação da Caatinga, v. 2, n. 1, p. 3-74, 2003.

REBOITA, M.S.; GAN, M.A.; ROCHA, R.P.; AMBRIZZI, T. Regimes de precipitação na América do Sul: Uma revisão bibliográfica. Revista Brasileira de Meteorologia, v. 25, n. 2, p. 185-204, 2010.

REBOITA, M.S.; DIAS, C.G.; DUTRA, L.M.D.; ROCHA, R.P.; LLOPART, M. Previsão climática sazonal para o Brasil obtida através de modelos climáticos globais e regional. Revista Brasileira de Meteorologia, v. 33, n. 2, p. 207224, 2018.

SAHA, S.; MOORTHI, S.; WU, X.; WANG, J.; NADIGA, S.; TRIPP, P.; BEHRINGER, D.; HOU, Y.T.; CHUANG, H.Y.; IREDELL, M.; EK, M.; MENG, J.; YANG, R.; MENDEZ, M.P.; DOOL, H.V.D.; ZHANG, Q.; WANG, W.; CHEN, M. \& BECKER, E. The NCEP climate forecast system version 2. Journal of Climate, v. 27, n. 6, p. 2185-2208, 2014.

SILVA, M.C.L. Modelagem Climática Regional do Jato de Baixos Níveis a Leste dos Andes e Validação. Dissertação de Mestrado, Programa de Pós Graduação em Meteorologia, Universidade de São Paulo, São Paulo, 2006.
SOUZA, E.B.; CARMO, A.M.; MORAES, B.C.; NACIF, A.; FERREIRA, D.B.; ROCHA, E.J.; SOUZA, P.O. Sazonalidade da precipitação sobre a Amazônia Legal brasileira: Clima atual e projeções futuras usando o modelo Regcm4. Revista Brasileira de Climatologia, v. 18, n. 12, p. 293306, 2016.

TAYLOR, K.E. Summarizing multiple aspects of model performance in a single diagram. Journal of Geophysical Research: Atmospheres, v. 106, n. D7, p. 7183-7192, 2001.

VANZOLINI, P.E. Paleoclimas e especiação em animais da América do Sul tropical. Estudos Avançados, v. 6, n. 15, p. 41-65, 1992.

WILKS, D.S. Statistical Methods in the Atmospheric Sciences. Amsterdam: Academic Press, p. 676, 2011.

ZHONG, Z.; HU, Y.; MIN, J.; XU, H. Numerical experiments on the spin-up time for seasonal-scale regional climate modeling. Journal of Meteorological Research, v. 21, n. 4, p. 409-419, 2007.

\section{Endereços de Internet}

Instituto Nacional de Meteorologia, http://inmet.gov.br/portal/ index.php? $\mathrm{r}=$ clima/desvioChuvaTrimestral.

License information: This is an open-access article distributed under the terms of the Creative Commons Attribution License (type CC-BY), which permits unrestricted use, distribution and reproduction in any medium, provided the original article is properly cited. 